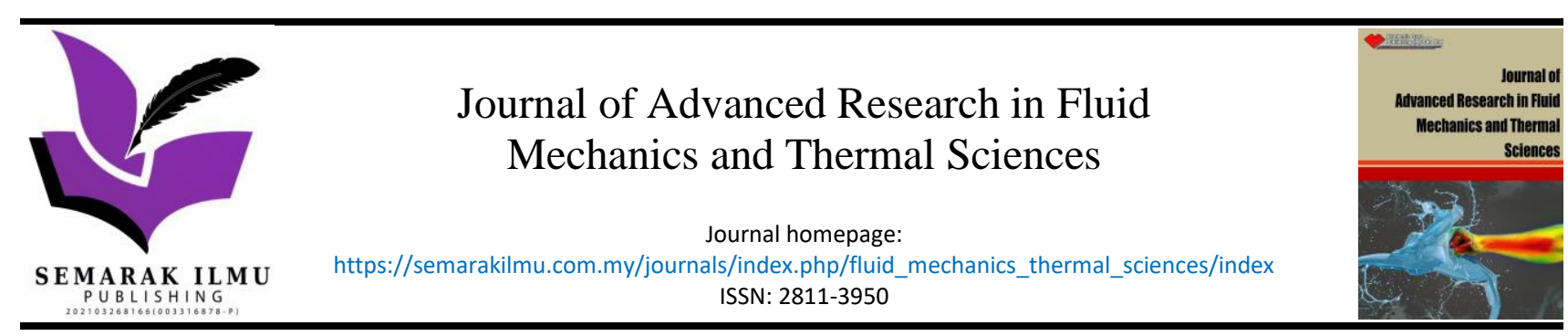

\title{
The Optimisation of Aerodynamic Performance Enhancement of a Flapping Wing using Response Surface Methodology
}

\author{
Hamid Yusoff ${ }^{1}$, Aliff Farhan Mohd Yamin ${ }^{2}$, Siti Nur Amalina Mohd Halidi' ${ }^{2}$, Nor Suhada Abdullah², \\ Halim Ghafar', Shafiq Suhaimi ${ }^{3}$, Koay Mei Hyie ${ }^{3}$, Wan Mazlina Wan Mohamed ${ }^{3,4, *}$ \\ Advanced Mechanics Research Group, Faculty of Mechanical Engineering, Universiti Teknologi MARA Cawangan Pulau Pinang, Malaysia \\ Faculty of Mechanical Engineering, Universiti Teknologi MARA Cawangan Pulau Pinang, Malaysia \\ School of Mechanical Engineering, College of Engineering, Universiti Teknologi MARA Shah Alam, 40450 Shah Alam, Selangor, Malaysia \\ Malaysia Institute of Transport (MITRANS), Universiti Teknologi MARA, 40450 Shah Alam, Selangor, Malaysia
}

ARTICLE INFO

\section{Article history:}

Received 28 July 2021

Received in revised form 11 December 2021

Accepted 19 December 2021

Available online 19 January 2022

Keywords:

Aerodynamics; Micro Air Vehicles; Flapping Wing; Response Surface Methodology

\section{ABSTRACT}

Flapping-Wing Micro Air Vehicles (FW-MAVs) are small hand-held flying vehicles that can manoeuvre in constrained space owing to their lightweight, low aspect ratio and can fly in a low Reynolds number environment. Among all wing types, flapping wings have the most potential in fulfilling this flight requirement since it offers good agility in confined spaces. However, the design problem for the flapping wings lies in lift generation and figuring out the main factors that play the biggest role in generating lift. In the present work, the effects of design parameters such as camber, velocity and frequency of the flapping wing have been investigated using the response surface methodology (RSM). The standard quadratic model of RSM called Central Composite Design (CCD) was utilized to identify and optimize the flapping wing. The experimental results were found to be in good agreement with the predicted outcomes. Out of 20 experimental trials determined by CCD, the optimum camber, velocity, and frequency were found to be $15 \%, 4.29 \mathrm{~m} / \mathrm{s}$ and $9 \mathrm{~Hz}$ respectively. The experimental results in this study are reasonable and accurate and can be used for describing the aerodynamic parameters. This study also established the usefulness of RSM in optimizing the aerodynamic properties for flapping wing mechanism.

\section{Introduction}

Micro air vehicles (MAVs) are defined as an unmanned aircraft that have a size that is smaller than $15 \mathrm{~cm}$ and have a take-off weight of less than $10 \mathrm{~g}$ [1]. Recent developments of MAV technology have shown potential in both military and civilian use. These examples of the uses of a MAV are military reconnaissance, crowd control, traffic management, survivor search, high-risk indoor inspection and other similar difficult tasks. MAVs can be filled under three categories; the first is Fixed-wing MAV, which can be used for long outdoor missions [2]. The second is Rotary-wing MAV, which usually used for short-distance outdoor missions and missions that requires hover [3]. The

\footnotetext{
* Corresponding author.

E-mail address: wmazlina@uitm.edu.my
}

https://doi.org/10.37934/arfmts.91.1.6982 
third is Flapping-wing MAV, which can be used for indoor missions [4]. Even though MAVs are designed to operate under difficult circumstances, they have several limitations. The conventional fixed and rotary MAVs have poor performance capabilities at low Reynolds number (low flight airspeed $<10 \mathrm{~m} / \mathrm{s}$ ) in comparison to its performance at higher Reynolds numbers [5] Due to its wingspan and surface area, the fixed-wing MAVs have lower agility in avoiding indoor obstacles, and due to its high-frequency rotation makes Rotary Wing-MAV noisy. Operating in Low Reynolds number also makes Rotary Wing-MAV has lower efficiency [6].

In contrast to MAV, a flapping flight offers good maneuvering and agility in confined spaces and can be considered as an alternative to conventional MAVs. However, one of the problems of designing a flapping wing is that the examples of man-made flapping wings are limited. Because of that, engineers turn to nature for inspiration. In nature, there are three main wing types: bird wings [7], insect wings [8] and bat wings. The main focus of this paper will be on bat wings.

Several studies have been carried out to understand aerodynamics associated with the flapping mechanisms. They have established that Flapping wings derive their lift from flapping motion of the wing in contrast to the conventional MAV's which utilize the forward airspeed of the aircraft [9]. This means that the lift and thrust the wing does not rely on the speed of the incoming air but rather depends on the motion of the wing itself. Review by Ho et al., [5] and Shyy et al., [10] provides excellent information on the advances in the aerodynamic performance of flapping-wing micro air vehicle (FW-MAV). A flapping wing is successful when the wing can mimic the motions of an actual wing found in nature while provides the maximum degrees in of freedom [11]. However, the difficulty of a flapping wing lies in keeping the symmetry of the flapping motion and the wide-angle of the flapping motion [12]. The lightweight and low aspect ratio FW-MAV is designed to fly under low Reynolds number conditions, which makes it easily susceptible to environmental airstreams. Therefore, there is a need for an improved wing having excellent aerodynamic features, which can achieve stable and successful flight at low Reynolds number.

To generate enough lift for a MAV to hover while moving at a slow forward speed is difficult, especially when the quasi-state aerodynamic theory is taken into consideration [8]. Bats and flying mammals however have solved the problem efficient low-speed flight by having thin and flexible wings. This is because bat wings have the capability of area expansion and contraction to produce a wing chamber shape that can adapt to undesirable gusty wind (Song et al., 2008). To produce a wing that can that is agile and efficient in a low-speed flight, FW-MAV designers take inspiration from nature where wings with a membrane skin allow the wing to be adaptable and change its camber. Despite the potential of membrane wings, there has yet a deep exploration about adaptive wing shape of a flexible wing membrane analysis. Most of the efforts that have been made instead are adopting camber wing shape in working MAVs [13]. It has been found that camber wing can provide additional enhancement of superior aerodynamic performance compared to the flat wing for the same design consideration. A previous investigation by the authors has also resulted in some interesting findings on utilizing adaptive camber wing [12]. Although several studies have been done on the cambered wing, these works mainly focused on fixed-wing MAVs only. It is not until recently that the aerodynamic advantages of a cambered wing are being explored in a flapping wing application [14]. Also, aerodynamic data related to different cambers in flapping flight are still limited. Major effects of camber on the aerodynamic performance of a flapping wing have been shown in a study done by Shkarayev et al., [15] by comparing the time average lift and thrust of a wing different camber and a rigid flat wing. In reality, it is not just one factor that played a role in the aerodynamic performance of the wing the combination of factors. There has yet a work done that investigates how much each factor contributes to the aerodynamic performance of a bat based flapping wing. 
Optimization of the aerodynamic parameters is essential to ensure the lifespan and determining the efficiency of operation. Several researchers have used different statistical tools like gradientbased optimization method, numerical optimization, Hooke Jeeves, and least square non-linear methods to optimize either the propulsive efficiency or enhance the lift generation. However, mostly the kinematic or shape/geometric parameters were under consideration in their studies [16]. As per our knowledge, no such optimization studies have been carried out to improve the aerodynamics based on camber, velocity, and frequency. Among numerous existing optimization methods, Response Surface Methodology (RSM) has been widely used in many applications [17]. There is evidence of its successful usage in applications ranging from heat transfer in electronic applications to wastewater treatment and environmental landfill site studies. RSM is a procedural statistic collection used as a global optimization method that derives from regression analysis and statistical design of experiments. RSM is a consecutive experimentation strategy for building and optimizing the empirical model by determining relationships between different design parameters and responses with the desired design criteria through empirical modelling. It can also be used for determining the significance of the different parameters on the responses. This means that RSM is useful for optimizing design parameters on desired value and response function and modelling and analysis which responses is affected by which design parameters (Ghommem, $\mathrm{M}$ et al., 2010). The needed response to the independent input model was achieved through experiment design and regression analysis application.

This study will focus on investigating camber of a flapping wing that will be used for MAV by expanding on the work done by Shkarayev et al., 2010 and investigating the effects of different camber of a wing. This was done by an experimental evaluation of the aerodynamic performance of a cambered wing compared to a flat wing. The aerodynamic performance was measured by testing the wings for time-averaged lift and drag generated with changing flapping frequency, free stream velocity, and angle of attack. These parameters were controlled by a flapping mechanism that was integrated with an electronic control system that was previously developed by the author in a past study. RSM was used as a basis for a systematic experimental design to investigate the effects of design parameters on the aerodynamic efficiency $\left(\varepsilon_{\text {aero }}\right)$ of the flapping wing. Additionally, the present study was extended to the quadratic model of RSM called Central Composite Design (CCD) to identify and optimize the flapping wing based on several factors like flapping frequency and free stream velocity.

\section{Methodology}

\subsection{Experimental Setup and Procedure}

In this study, a novel flapping wing was tested for its lift and drag generation using a strain gauge sensor and an open airflow chamber. The airflow chamber (Figure 1a) uses a propeller at the rear of this chamber generates the requisite wind velocity, which was controlled by a digital controller. Undesired turbulence was avoided by storing the intake air in a reservoir before being channeled out to the test chamber. To avoid undesired turbulence, intake air is stored in a reservoir, before being channeled out and a digital controller is used to control the airspeed. The test chamber is an open section with non-return airflow with a dimension of $1 \times 1 \mathrm{ft}(0.3 \times 0.3 \mathrm{~m})$. The test chamber is capable of airspeed of 0.5 to $30 \mathrm{~m} / \mathrm{s}$. The uniformity of free stream velocity was verified by testing the turbulence level of axial flow direction was using Laser Doppler Anemometry (LDA) which was rated at $0.3 \%$.

A high precision Deltalab strain gauge sensor was used to measure the generated lift and drag of the wing by attaching the sensors to the flapper system using an intermediate stand. The initial 
system has been wired and configured to provide measurements for lift, drag and moment. The measurement of the lift, drag, and moment have based the displacements of four rigid beams with strain gauges attached to them. These beams are then subjected to bending and torsional loads that came from the generated aerodynamic forces. The test model will be placed perpendicular to the flow direction and the displacement of the model will insignificant. The strain sensor has a precision of $0.3 \%$ maximum error out of $5 \mathrm{~N}$ full-scale force.

The data acquisition system was used for this study is a Kyowa DAQ-type of PCD 300A model data acquisition where the system can sample up to 5000 samples per second for every channel input. The user interface (UI) of the DAQ device that was used in is the LabView 6.0 software and the raw data that was collected was exported to Microsoft Excel. The DAQ also has an 8-bit resolution. The PCD300A was calibrated under default channel conditions settings with a $10000 \mu \mathrm{m} / \mathrm{m}$ range, $1.67-$ calibration factor, and zero offset value (refer Figure $1 \mathrm{~b}$ for setting up). Low pass Butterworth filter with a cut-off frequency of $5 \mathrm{~Hz}$ and a second-order iterative process was used to smooth the raw data. A total of 40,000 data points was collected for every point test condition, which was integrated into lift and coefficients of drag timed average value, using the relations CL avg (Eq. 1) and CD avg (Eq.2) respectively.

$C_{L \text { avg }}=\frac{L_{\text {avg }}}{0.5 \rho V_{\infty}^{2} S}$

$C_{D \text { avg }}=\frac{D_{\text {avg }}}{0.5 \rho V_{\infty}^{2} S}$

where $L_{\text {avg }}$ - the average lift force, $D_{\text {avg }}$ - the average drag force, $S$ - wing platform area, $V_{\infty}-$ forward flight speed and $\rho$-air density.

To accurately determine the flapping frequency, an Electronic Control System (ECS) consisting of DC mini-motor with encoder, variable resistor, power supply, motor driver, microcontroller, and a personal computer with GUI (Graphical User Interface) that was built by the author for a previous study (refer to our previous work by Yusoff et al., 2015), which is better than previous ways of flapping wing frequency measurement because the ECS can reduce the relative error from $25-35 \%$ to $0.4-1.8 \%$. Following the work of Shkarayev et al., 2010, five wind tunnel models having 6, 9, 12, and $15 \%$ camber were developed. The same wing design as used in our previous work (Yusoff et al., 2015), was utilized for the current study. These wings had the same chord length c, wing area $A$, and thickness $t$ (see also Figure 1d-1f). Table 1 shows the physical features for wings with different camber percentage. Figure $(1 a-1 b)$ shows the experiment and schematic setup of the present study.

\section{Table 1}

The series of wing model geometry

\begin{tabular}{llllll}
\hline Camber, $(\mathrm{h} / \mathrm{c}) \%$ & 6 & 9 & 12 & 15 & Flat \\
Wing area A, m2 & 0.013 & 0.013 & 0.013 & 0.013 & 0.013 \\
Chord length c, $(\mathrm{mm})$ & 0.08 & 0.08 & 0.08 & 0.08 & 0.08 \\
Camber height $\mathrm{h},(\mathrm{mm})$ & 4.8 & 7.2 & 9.6 & 12 & - \\
Thickness t, $(\mathrm{mm})$ & 0.35 & 0.35 & 0.35 & 0.35 & 0.35 \\
\hline
\end{tabular}




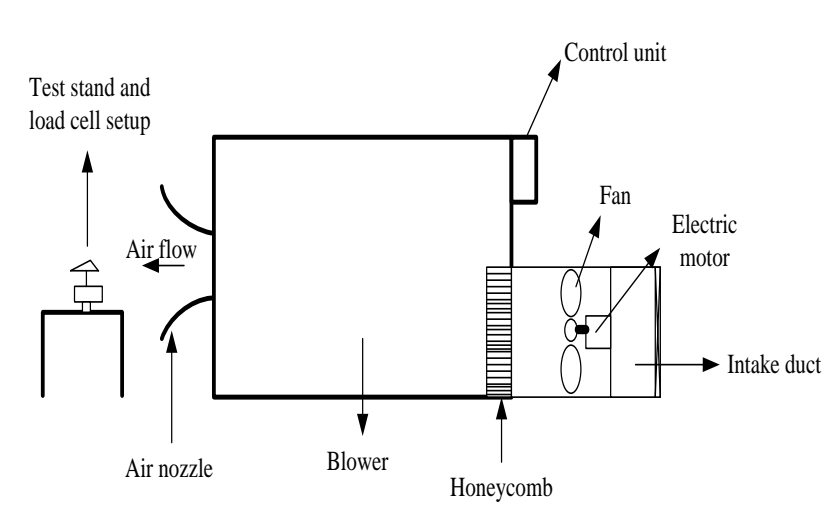

(a)

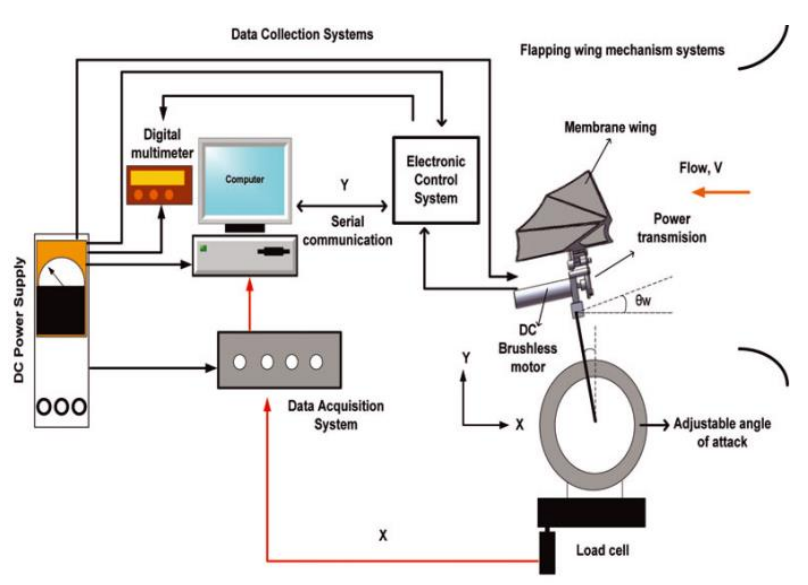

(b)

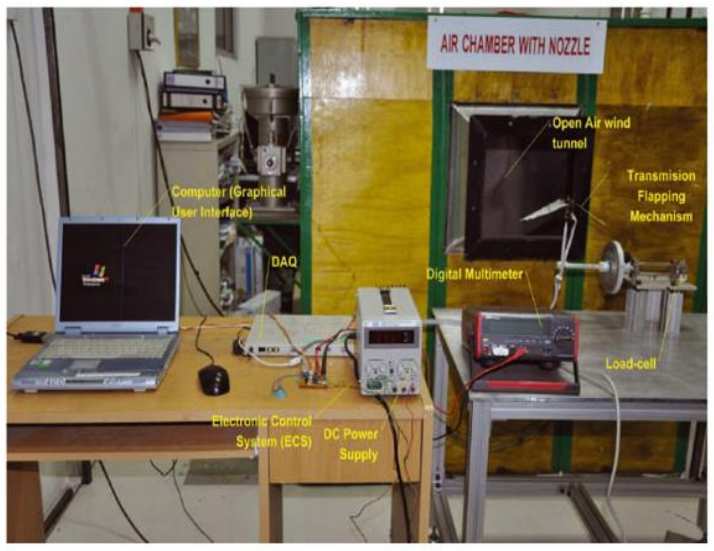

(c)

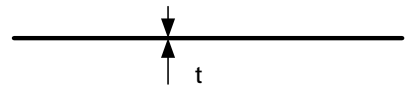

(d)

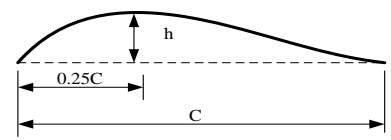

(f)

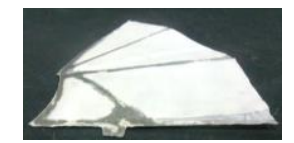

(e)

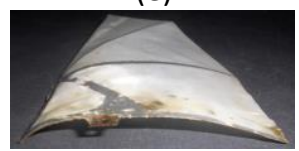

(g)

Fig. 1. (a) The apparatus of experimental (b) schematic of experimental setup by Yusoff et al., [8] (c) schematic of air chamber design study (d)-(e) schematic of flat and photo wing, and (f)-(g) schematic of camber and photo wing

Each model was mounted in the wind tunnel and tested over a range of free-stream velocity $(V)$ from 1 to $7 \mathrm{~m} / \mathrm{s}$, corresponding to Reynolds number ranging from 3600 to 25,200 and flapping frequency $(\mathrm{F})$ of 4 to $9 \mathrm{~Hz}$. The pitch angle of the flapping axis $(\vartheta w)$ was varied concerning the angle of attack ranging from 00 to 500 and free stream velocity by adjusting the test stand of the flapper system.

\subsection{Performance Characteristics}

Shape, wing size, conditions of the air and velocity of flights are the factors that influence both lift and drag, where lift acts upon the body in a direction that is perpendicular to the flight path while drag acts on the body in a direction that is along the flight path. In aerodynamics, the aerodynamic 
performance (or aerodynamic efficiency, $\varepsilon_{\text {aero }}$ ) characteristics and is defined as the ratio between the lift generated and the drag where:

$\varepsilon_{\text {aero }}=\frac{C_{L}}{C_{D}}=\frac{L}{D}$

For a wing, the high aerodynamic performance can be achieved when the lift-to-drag ratio is maximized. The higher the ratio is the better the flight performance because the lift needed to be generated by an aircraft depends on the weight of the aircraft and higher lift with lower drag means that it is possible for the flight to have better fuel economy, better climb performance, and better glide ratio.

According to Ho et al., 2003, there are two flapping flight regimes, which are quasi-steady flight and unsteady flight where the quasi-steady flight is when the wing is hardly flapping or flapping at a very low frequency. This means that the speed wing tip is lower than the flight velocity. This type of regime is common in larger birds like eagles and seagulls where their flight behavior tends to look like a fixed-wing flight as these birds soar more than flap their wings during flight. The second flight regime is the unsteady state regime where the flapping frequency is higher and the wing tip speed faster than the forward velocity of the flight. This type of flight regime is more common in smaller birds and insects. The assumptions made in a quasi-steady flight cannot be applied to an unsteady state flight regime due to the unsteady nature of the flight regime. In this study, the flapping flight is characterized by advance ratio ' $\mathrm{J}$ ', which is a non-dimensional parameter that is the ratio of forwarding flight speed to the wing tip velocity during flight. Advance ratio is usually common in characterizing the aerodynamics of a rotary-wing flight but this it is also applicable to a flapping-wing flight. The advance ratio, 'J' can be expressed as:

$J=\frac{V_{\infty}}{2 f b \Phi}$

where $f$ is the wing flapping frequency, and $\phi$ is the peak-to-peak displacement of the wingtip during the flapping flight.

\subsection{Experimental Conditions and Plan}

In this study, the parameters that are used as design variables are the camber, velocity, and frequency because of the strong influence these parameters have on the aerodynamic efficiency $\left(\varepsilon_{\text {aero }}\right)$. A type of RSM named central composite design (CCD) was used to determine experimental plan setting the effect the aerodynamic efficiency $\left(\varepsilon_{\text {aero }}\right)$. The factors part of CCD is a full factorial design that has all the combination of factors that has two levels, which are: high, +1 and low, -1 . These factors are made of eight-star points and the six central points are the middle points between high and low levels are coded as level 0 . The type of design that was used was face centered CCD where the $\alpha$ value of 1 correlate to the star points that are at the face of the cube portion on the design. The designation of the different design parameters and the different levels of these design parameters were shown in Table 1. Table 2 shows that the Angle of Attack, AoA was restricted to $10^{\circ}$. In this study, at the before mentioned conditions, 20 experiments were made based on the facecentered CCD where the response variable that was investigated was the $\varepsilon_{\text {aero. }}$. To achieve a more accurate result, each combination of experiments was repeated three times. 


\section{Table 2}

\begin{tabular}{lllll}
\multicolumn{4}{l}{ The scheme of design parameters and their levels } \\
\hline \multirow{2}{*}{ Symbol } & Factor & Unit & Levels \\
\cline { 3 - 5 } & & Low (-1) & High (+1) \\
\hline A & Camber & - & 0 & 15 \\
B & Velocity & $\mathrm{m} / \mathrm{s}$ & 1 & 7 \\
C & Frequency & $\mathrm{Hz}$ & 4 & 9
\end{tabular}

There are several factors inside a CCD, these factors are the $2 \mathrm{k}$ with $\mathrm{nF}$ factorial are points with all possible combination of the minimum and maximum values of the control parameters. The second the $2 \mathrm{k}$ or otherwise known as star runs which is one of the parameters that have the minimum or maximum value, and all other parameters have their nominal value. The final factor is the $\mathrm{nC}$ center runs where all controls parameters are set to their nominal values. In the CCD equation where CCD $=2 \mathrm{k}+2 \mathrm{k}+8$ where $\mathrm{k}$ is the number of factors. 20 experimental were improved with eight replications at the design center to evaluate pure error [18]. Eq. (4) shows the quadratic model used to estimate the optimal point:

$Y=\beta_{o}+\sum_{j=1}^{k} \beta_{j} X_{j}+\sum_{j=1}^{k} \beta_{j j} X_{j}^{2}+\sum_{i} \sum_{<j=2}^{k} \beta_{i j} X_{i} X_{j}+e_{i}$

where $Y$ is the response; $X_{i}$ and $X_{j}$ are the variables; $B_{0}$ is a constant coefficient; $B_{j}, b_{j j}$ and $B_{i j}$ are the interaction coefficients of linear, quadratic and second-order terms, respectively; $k$ is the number of studied factors, and $e_{i}$ is the error.

The quality and the lack of fit of the polynomial model was identified using the coefficient of determination (R2) and valuating the variables and the interaction between these variables was determined using the P-value associated with 95\% confidence level. Adequate Precision, probability value (ProbNF) and the Fisher evaluation ratio (F-value) were used to measure the model significance and the model's adequacy. The value of response variable, in this case, the aerodynamic efficiency $\left(\varepsilon_{\text {aero }}\right)$, was identified at maximizing targeted response, as such in the most desired ranges.

\section{Results and Discussion}

\subsection{Unsteady Effect of Lift and Drag Performance}

The flow around a flapping wing can be considered quasi-steady when $\mathrm{J}>1.0$, while $\mathrm{J}<1.0$ corresponds to unsteady state regime. For ease of comparison, the exponential decay is also plotted on the same graph for the flat and $15 \%$ camber wings as shown in Figure 2. Our experimental results confirm that the present set up belonged to the region of unsteady and quasi-steady flow. Based on these results, for an advance ratio lesser than 1 , the value of $C_{L}$ avg and $C_{D}$ avg for all the wings increased exponentially. On the other hand, when the advance ratio increased, $C_{L}$ avg and $C_{D}$ avg values decreased rapidly which can jeopardize the aerodynamic benefits of flapping wings. It has been established that under quasi-steady regime with relatively large values of $\mathrm{J}$, there is virtually limited or zero aerodynamic benefits to $C_{L \text { avg }}$ and $C_{D}$ avg.

Therefore, to compare the results of $C_{L \text { avg }}$ and $C_{D}$ avg for every increment in camber wing, a flat wing was utilized. It was found that the maximum camber wing $15 \%$ provided the best values for lift and drag coefficients. This was because, as the camber increases, the lift force also increases. For this configuration, both the $C_{L \text { avg }}$ and $C_{D}$ avg showed increased aerodynamic benefits. The $C_{L \text { avg }}$ value was 
enhanced by 2 3 times and $C_{D}$ avg demonstrated $0.5^{\sim} 0.7$ times improvement, in comparison to the flat wing performance for an advance ratio less than 1 . However, when the advance ratio was more than 1 (i.e., quasi-steady) the amount of lift increment for camber 15\% did not show any significant benefits. The increase in lift was only around 0.5 0.7 times and for drag the increment was just $0.3 \sim 0.5$ times, much lesser than the case with lower values of advance ratio. This is indicative of the fact that the flapping wings have better aerodynamic benefits under unsteady flow regimes.

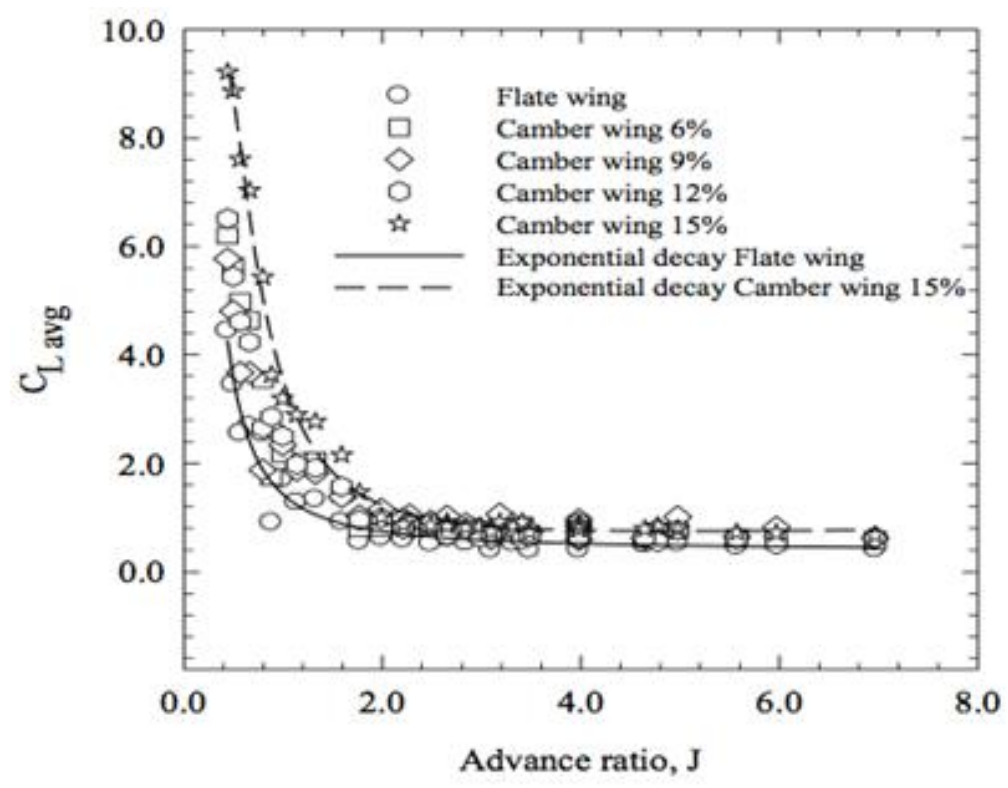

(a)

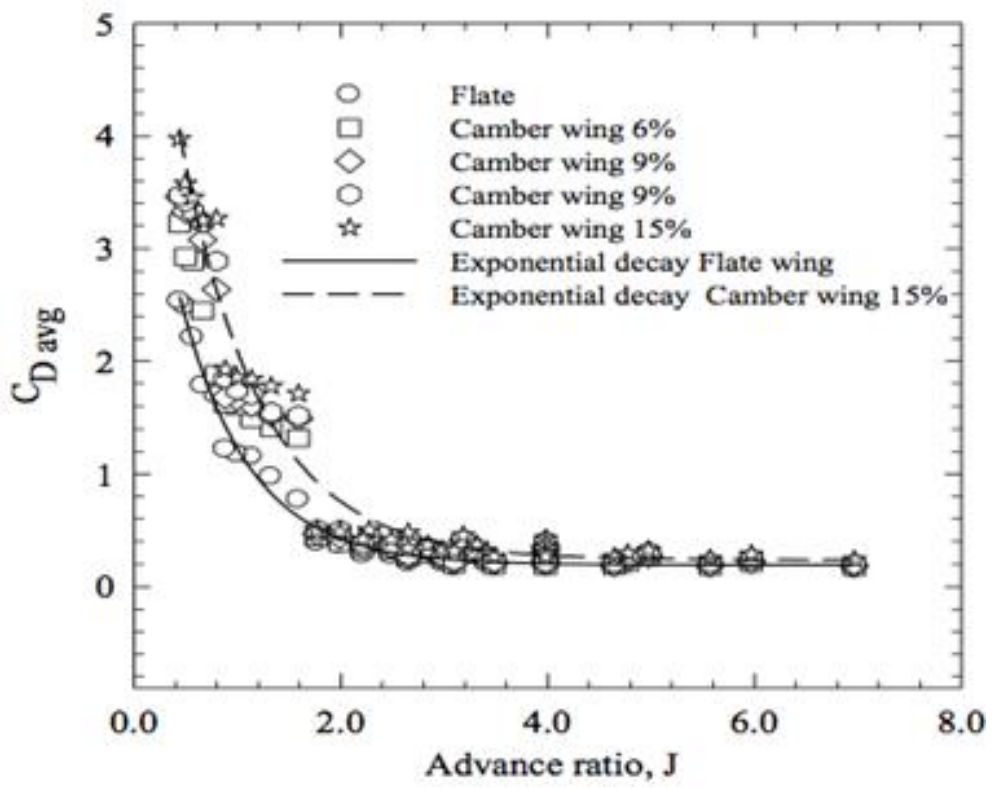

(b)

Fig. 2. (a) $C_{L \text { avg }}$ and (b) $C_{D \text { avg }}$ respect to advance ratio (J)

\subsection{Result of DOE Analysis}

Table 3 shows the result for each wing $\varepsilon_{\text {aero }}$ evaluation. The compatibility of the quadratic model with the experimental data was tested with a significance test of the individual model coefficients and a test for lack of fit. ANOVA analysis was used to provide a summary of the performed test. 


\section{Table 3}

Design of experimental matrix and results for the $\varepsilon_{\text {aero }}$ characteristics

\begin{tabular}{lllll}
\hline & \multicolumn{2}{l}{ Design parameters } & \multicolumn{2}{l}{ Experimental result } \\
\cline { 2 - 4 } Exp. no. & A & B & C & Eaero $=C_{\text {L avg }} / C_{\text {d avg }}$ \\
\cline { 2 - 4 } & Camber $\%$ & Velocity & Frequency & 2.21439 \\
2 & 15 & 7 & 9 & 1.45403 \\
3 & 0 & 4 & 6.5 & 2.02907 \\
4 & 7.5 & 4 & 6.5 & 2.32716 \\
5 & 15 & 1 & 4 & 2.02734 \\
6 & 7.5 & 4 & 6.5 & 3.434 \\
7 & 7.5 & 4 & 9 & 2.04286 \\
8 & 7.5 & 4 & 6.5 & 1.77669 \\
9 & 0 & 7 & 9 & 1.62451 \\
10 & 0 & 1 & 9 & 1.69793 \\
11 & 7.5 & 7 & 6.5 & 2.28932 \\
12 & 15 & 7 & 4 & 2.27933 \\
13 & 15 & 4 & 6.5 & 0.678571 \\
14 & 0 & 1 & 4 & 1.27952 \\
15 & 0 & 7 & 4 & 2.50435 \\
16 & 15 & 1 & 9 & 2.0539 \\
17 & 7.5 & 4 & 6.5 & 0.607273 \\
18 & 7.5 & 1 & 6.5 & 1.6754 \\
19 & 7.5 & 4 & 4 & 2.01678 \\
20 & 7.5 & 4 & 6.5 & 2.07091 \\
\hline & 7.5 & 4 & & \\
\hline
\end{tabular}

\subsection{ANOVA Analysis}

Table 4 shows the result of the ANOVA $\varepsilon_{\text {aero }}$ quadratic model. The results in table 4 show that the "Prob. $>F$ " value for this model is less than 0.05 , which means that the $\alpha=0.05$, or $95 \%$ confidence. This shows that the model is statically significant, and the model has a significant impact on the response. The same can also be said to the main effect of factor $A$ (camber), factor $C$ (frequency), the second-order effect of factor B (velocity), and the second-order effect of factor $C$ (frequency) are significant model terms. The results also show that the double effect of factor $A$ (camber) and factor $B$ (velocity) shows that the $\varepsilon_{\text {aero }}$ is derived from the wing module under constant AoA. All other model terms are insignificant as their "Prob. $>F$ " value is greater than 0.05 and can be removed.

The quadratic model of the $\varepsilon_{a e r o}$ was adjusted and the insignificant terms were eliminated through the elimination process. Table 5 shows the ANOVA table for the $\varepsilon_{\text {aero }}$ reduced quadratic model where the result had shown that the model is still significant for "Prob. F)" less than 0.005 statuses. Also, an important coefficient in the ANOVA table is the R2 coefficient where the R2 is defined as the ratio of the explained variation to the total variation. $R 2$ is measured in terms of the degree of fit and the results in table 5 have shown that theR2 value for the reduced model is at a level of over 0.75 where the model explained $75 \%$ of the data variability. This means that the model adequately explained the relationship between the independent factors and $\varepsilon_{\text {aero, }}$ which is the response. The model also has an average prediction error of above 4 where it means that the model has an adequate precision. The model also has good adequate discrimination because the value of the ratio is more than 4 . 
Table 4

ANOVA table for the $\varepsilon_{\text {aero }}$ (before elimination)

\begin{tabular}{|c|c|c|c|c|c|}
\hline Source & Sum of squares & $\begin{array}{l}\text { Degree of } \\
\text { freedom }\end{array}$ & $\begin{array}{l}\text { Mean } \\
\text { square }\end{array}$ & F-value & Prob $>\mathrm{F}$ \\
\hline Model & 6.13 & 9 & 0.68 & 5.63 & 0.0062 \\
\hline$A$ & 2.31 & 1 & 2.31 & 19.05 & 0.0014 \\
\hline B & 0.23 & 1 & 0.23 & 1.90 & 0.1983 \\
\hline C & 1.09 & 1 & 1.09 & 9.02 & 0.0133 \\
\hline$A 2$ & $6.52 \mathrm{E}-003$ & 1 & $6.52 \mathrm{E}-003$ & 0.054 & 0.8211 \\
\hline B2 & 1.60 & 1 & 1.60 & 13.22 & 0.0046 \\
\hline $\mathrm{C} 2$ & 1.12 & 1 & 1.12 & 9.29 & 0.0123 \\
\hline$A B$ & 0.15 & 1 & 0.15 & 1.21 & 0.2977 \\
\hline$A C$ & 0.22 & 1 & 0.22 & 1.86 & 0.2029 \\
\hline$B C$ & 0.061 & 1 & 0.061 & 0.51 & 0.4926 \\
\hline Residual & 1.21 & 10 & 0.12 & & \\
\hline Lack of fit & 1.21 & 5 & 0.24 & 611.65 & $<0.0001$ \\
\hline Pure error & $1.976 \mathrm{E}-003$ & 5 & $3.951 E-004$ & & \\
\hline Cor. Total & 7.34 & 19 & & & \\
\hline \multicolumn{3}{|c|}{ Standard deviation $=0.35$} & \multicolumn{3}{|l|}{$\mathrm{R} 2=0.8352$} \\
\hline \multicolumn{3}{|c|}{ Mean $=1.90$} & \multicolumn{3}{|c|}{$\mathrm{R} 2$ adjusted $=0.6869$} \\
\hline \multicolumn{3}{|c|}{ Coefficient of variation $=18.27$} & \multicolumn{3}{|c|}{ Adequate precision $=10.139$} \\
\hline \multicolumn{6}{|c|}{ Predicted residual error of sum of squares (PRESS) $=9.27$} \\
\hline
\end{tabular}

Table 5

ANOVA table for the $\varepsilon_{\text {aero }}$ (after elimination)

\begin{tabular}{|c|c|c|c|c|c|}
\hline Source & $\begin{array}{l}\text { Sum of } \\
\text { squares }\end{array}$ & $\begin{array}{l}\text { Degree of } \\
\text { freedom }\end{array}$ & $\begin{array}{l}\text { Mean } \\
\text { square }\end{array}$ & F-value & Prob $>\mathrm{F}$ \\
\hline Model & 5.69 & 5 & 1.14 & 9.67 & 0.0004 \\
\hline A & 2.31 & 1 & 2.31 & 19.57 & 0.0006 \\
\hline B & 0.23 & 1 & 0.23 & 1.95 & 0.1842 \\
\hline C & 1.09 & 1 & 1.09 & 9.27 & 0.0087 \\
\hline B2 & 1.95 & 1 & 1.95 & 16.57 & 0.0011 \\
\hline $\mathrm{C} 2$ & 1.23 & 1 & 1.23 & 10.48 & 0.0060 \\
\hline \multicolumn{6}{|l|}{ Residual } \\
\hline Lack of fit & 1.65 & 9 & 0.18 & 463.18 & $<0.0001$ \\
\hline Pure error & $1.976 \mathrm{E}-003$ & 5 & $3.951 \mathrm{E}$ & & \\
\hline Cor. Total & 7.34 & 19 & & & \\
\hline \multicolumn{3}{|c|}{ Standard deviation $=0.34$} & \multicolumn{3}{|c|}{$\mathrm{R} 2=0.7755$} \\
\hline \multicolumn{3}{|c|}{ Mean $=1.90$} & \multicolumn{3}{|c|}{ R2 adjusted $=0.6953$} \\
\hline \multicolumn{3}{|c|}{ Coefficient of variation $=18.02$} & \multicolumn{3}{|c|}{ Adequate precision $=11.031$} \\
\hline \multicolumn{6}{|c|}{ Predicted residual error of sum of squares (PRESS) $=4.59$} \\
\hline
\end{tabular}

The final quadratic model of response equation is achieved through a backward elimination process in terms of coded factors can be given as:

$\frac{L}{D}=1.98+0.48 A+0.15 B+0.33 C-0.76 B^{2}+0.62 C^{2}$

In terms of actual factors, the final quadratic models of response equation are as follow:

$$
\frac{L}{D}=3.25278+0.064016 A+0.74478 B+1.15966 C-0.086781 B^{2}+0.099371 C^{2}
$$


The model obtained above can be used to predict the aerodynamic efficiency $\left(\varepsilon_{\text {aero }}\right)$ within the limits of the factors studied. The normal probability plot of the residuals for the aerodynamic efficiency $\left(\varepsilon_{\text {aero }}\right)$ is displayed in Figure 3 . Notice that the residuals generally fall on a straight line implying that the errors are normally distributed. Further, it supports the adequacy of the leastsquare fit.

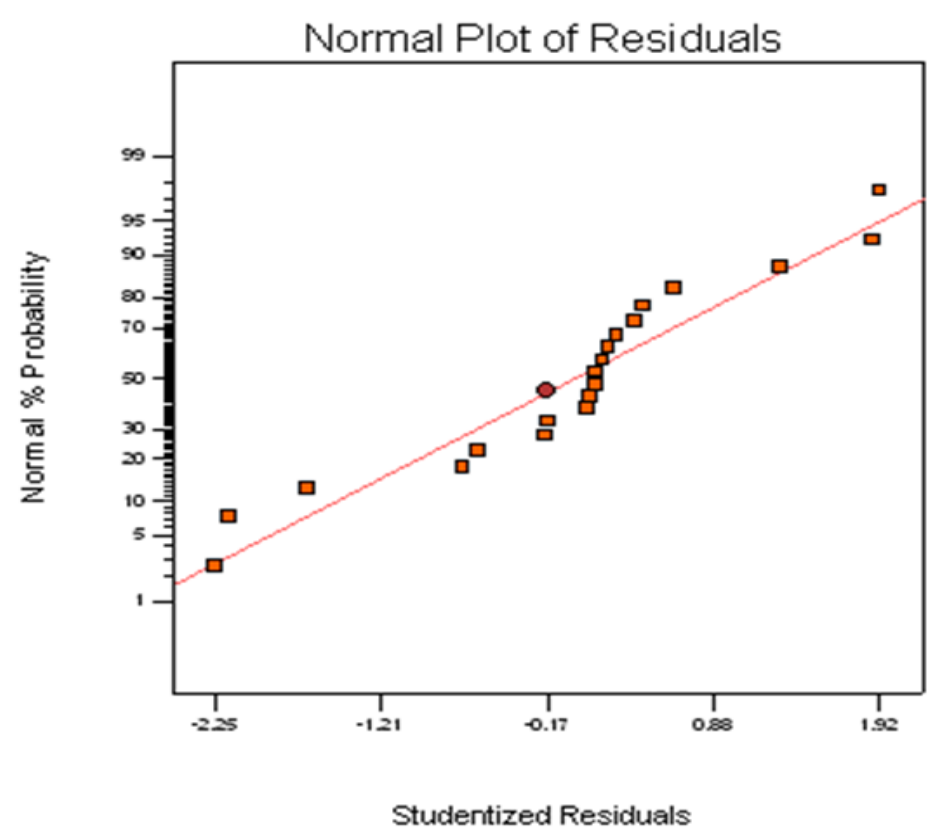

Fig. 3. Normal probability plot of the studentized residual for $\varepsilon_{\text {aero }}$

\subsection{Effect of Design Parameters on the $\varepsilon_{a e r o}$}

Perturbation plot is analyzed to further identify the most sensitive factors for aerodynamic efficiency $\left(\varepsilon_{\text {aero }}\right)$ as shown in (Figure 4$)$. Factor $A$ (camber) and factor $C$ (frequency) appeared to be the influential factors for enhancing aerodynamic efficiency $\left(\varepsilon_{\text {aero }}\right)$ instead of factor $B$ (velocity). However, the most influential factor is factor $A$ (camber). For example, the experimental results show that the lift-to-drag ratios measured at the same flow velocity conditions are shifted by the variation of the camber; this behavior conformed to the observation by Kim et al., 2006. This result was likely because cambered-wing could produce higher lift and thrust than the flat wing, which can perform strong leading-edge vortex (LEV) on the leading edge (LE) at the upper wing surface for a low-speed flight (i.e., unsteady flow), and the wing can sustain and maintain this reattachment for a long time.

The Design-Expert software was used to draw a 3D response and quadratic model counterplots to study the interactive relationship between the independent variable and responses. In Figure 5, two variables were found in the 3D surface response and counterplots to be varied in the experimental range. The other variables, however, were kept constant due to the level of sensitivity towards the responses that was shown in Figure 4. The factors that are kept at maximum values is the factor C (frequency) as can be seen in Figure 5. However, factor A (camber) and factor B (velocity) were varied because the perturbation plots, Figure 4 , shows that only two factors (camber and frequency) contributed significantly towards the aerodynamic efficiency (L/D). Based on Figure 5, the optimum condition was found to be at a maximum percentage of camber (15\%), with frequency $15 \mathrm{~Hz}$. 


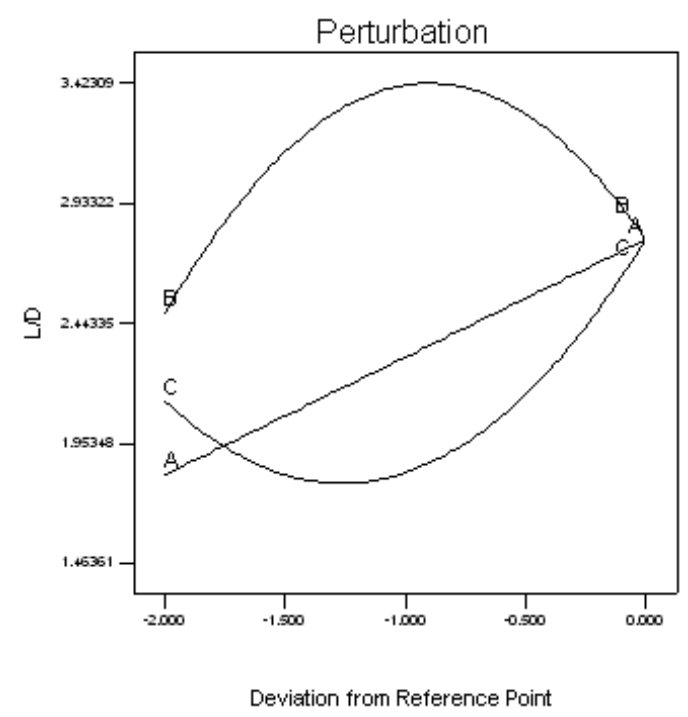

Fig. 4. Perturbation plot for aerodynamic efficiency $\left(\varepsilon_{\text {aero }}\right)$ (Note: $\mathrm{A}=$ Camber, $\mathrm{B}=$ Velocity, and $\mathrm{C}=$ Frequency)

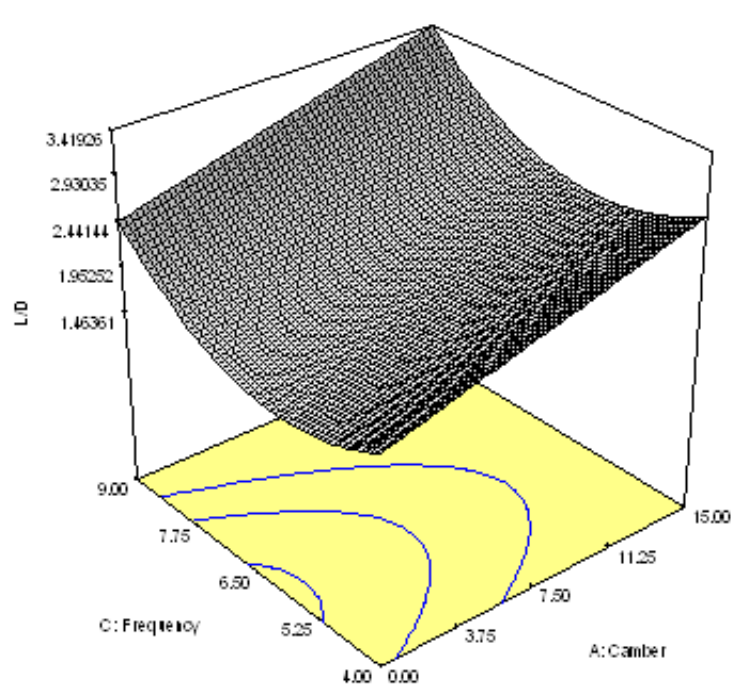

Fig. 5. 3D response surface for aerodynamic efficiency $\left(\varepsilon_{\text {aero }}\right)$

\subsection{Optimization of Design Parameters}

In this study, the main value of design parameters $(X)$ that was to be optimized was the maximum value of $\varepsilon_{\text {aero, }}$ where, in the optimization step in the software that was used, each design parameter (camber, velocity, and frequency) was set to "within the rage" and the response (aerodynamic efficiency, $\varepsilon_{a e r o}$ ) was set as "maximum". This is done to reach the highest performance, and this will also have made the program to generate a single number from the combination of each desirability and the program will also search so that the function is maximized. Table 6 shows the result for the established optimum working conditions and respective aerodynamic efficiency $\left(\varepsilon_{\text {aero }}\right)$. 
Table 6

Optimization results for aerodynamic efficiency $\left(\varepsilon_{\text {aero }}\right)$

\begin{tabular}{llllll}
\hline $\begin{array}{l}\text { Solution } \\
\text { no. }\end{array}$ & $\begin{array}{l}\text { Percentage of Camber } \\
(\%)\end{array}$ & $\begin{array}{l}\text { Velocity } \\
(\mathrm{m} / \mathrm{s})\end{array}$ & $\begin{array}{l}\text { Frequency } \\
(\mathrm{Hz})\end{array}$ & $\begin{array}{l}\varepsilon_{\text {aero }}=\mathrm{CL}- \\
\mathrm{avg} / \mathrm{CD}_{\text {avg }}\end{array}$ & Desirability \\
\hline 1 & 15.00 & 4.29 & 9 & 3.42309 & 0.984 \\
2 & 15.00 & 4.41 & 9 & 3.42182 & 0.981 \\
3 & 14.98 & 4.33 & 9 & 3.42143 & 0.980 \\
4 & 15.00 & 5.42 & 9 & 3.31165 & 0.725 \\
\hline
\end{tabular}

\subsection{Confirmation Experiments}

As shown in Table 6, a value of 3.42309 for aerodynamic efficiency $\left(\varepsilon_{\text {aero }}\right)$ was predicted according to the model under optimized operational conditions (percentage of camber $15 \%$, velocity $4.29 \mathrm{~m} / \mathrm{s}$, and frequency $9 \mathrm{~Hz}$ ). The desirability function value obtained for this optimum condition was 0.984 .

Analysis of the data in Table 7 has shown that the residual and percentage are small with only 5 to $2.5 \%$ of error between the experimental data and the calculated predicted value of $\varepsilon_{\text {aero, }}$ which means that the quadratic model was very accurate. Four confirmation experiments for $\varepsilon_{\text {aero }}$ made to validate this study's quadratic model where Table 7 has shown the confirmation data compared to the predicted values

Table 7

Confirmation Experiment

\begin{tabular}{lllllll}
\hline Exp no. & \multicolumn{5}{l}{ Design parameters } & \multicolumn{2}{l}{ Eaero } \\
\cline { 2 - 6 } & A & B & C & Exp. & Predicted & Error $(\%)$ \\
\hline 1 & 15.00 & 4.29 & 9 & 3.5302 & 3.42309 & 3.03 \\
2 & 15.00 & 4.41 & 9 & 3.4505 & 3.42182 & 0.83 \\
3 & 14.98 & 4.33 & 9 & 3.2506 & 3.42143 & 5.26 \\
4 & 15.00 & 5.42 & 9 & 3.2303 & 3.31165 & 2.51 \\
\hline
\end{tabular}

\section{Conclusions}

A systematic experimental design based on response surface methodology (RSM) was done to investigate the effects of different design parameters on a flapping wing, where the aerodynamic efficiency of the flapping wing with given design constraints was effectively predicted and optimized. The optimum conditions determined from the model for camber, velocity and frequency are $15 \%$, $4.29 \mathrm{~m} / \mathrm{s}$ and $9 \mathrm{~Hz}$ respectively. Also, the confirmation experiments showed that RSM is reasonably accurate, and it indicated only $5.06 \%$ deviation. Thus, this study was able to establish the usefulness of RSM in optimizing the aerodynamic properties for flapping wing mechanism.

\section{Acknowledgement}

The authors would like to acknowledge the financial support from Malaysia Institute of Transport (MITRANS) DeLima Grant (RS12021GRN18RN008) and Universiti Teknologi MARA, Cawangan Pulau Pinang.

\section{References}

[1] Ward, Thomas A., M. Rezadad, Christopher J. Fearday, and Rubentheren Viyapuri. "A review of biomimetic air vehicle research: 1984-2014." International Journal of Micro Air Vehicles 7, no. 3 (2015): $375-394$. https://doi.org/10.1260/1756-8293.7.3.375

[2] Hamizi, Ilya Bashiera, and Sher Afghan Khan. "Aerodynamics investigation of delta wing at low Reynold's number." CFD Letters 11, no. 2 (2019): 32-41. 
[3] Mohamed, Wan Mazlina Wan, Nirresh Prabu Ravindran, and Parvathy Rajendran. "A CFD Simulation on the Performance of Slotted Propeller Design for Various Airfoil Configurations." CFD Letters 13, no. 3 (2021): $43-57$. https://doi.org/10.37934/cfdl.13.3.4357

[4] Abas, Mohd Firdaus Bin, Azmin Shakrine Bin Mohd Rafie, Hamid Bin Yusoff, and Kamarul Arifin Bin Ahmad. "Flapping wing micro-aerial-vehicle: kinematics, membranes, and flapping mechanisms of ornithopter and insect flight." Chinese Journal of Aeronautics 29, no. 5 (2016): 1159-1177. https://doi.org/10.1016/i.cja.2016.08.003

[5] Ho, Steven, Hany Nassef, Nick Pornsinsirirak, Yu-Chong Tai, and Chih-Ming Ho. "Unsteady aerodynamics and flow control for flapping wing flyers." Progress in aerospace sciences 39, no. 8 (2003): 635-681. https://doi.org/10.1016/i.paerosci.2003.04.001

[6] Galiński, C. E. Z. A. R. Y., and R. Żbikowski. "Some problems of micro air vehicles development." Bulletin of the Polish Academy of Sciences: Technical Sciences (2007): 91-98.

[7] Yang, Wenqing, Liguang Wang, and Bifeng Song. "Dove: A biomimetic flapping-wing micro air vehicle." International Journal of Micro Air Vehicles 10, no. 1 (2018): 70-84. https://doi.org/10.1177/1756829317734837

[8] Karásek, Matěj, Florian T. Muijres, Christophe De Wagter, Bart DW Remes, and Guido CHE De Croon. "A tailless aerial robotic flapper reveals that flies use torque coupling in rapid banked turns." Science 361, no. 6407 (2018): 1089-1094. https://doi.org/10.1126/science.aat0350

[9] Gong, Chunlin, Jiakun Han, Zongjing Yuan, Zhe Fang, and Gang Chen. "Numerical investigation of the effects of different parameters on the thrust performance of three dimensional flapping wings." Aerospace Science and Technology 84 (2019): 431-445. https://doi.org/10.1016/j.ast.2018.10.021

[10] Shyy, Wei, Hikaru Aono, Satish Kumar Chimakurthi, Pat Trizila, C-K. Kang, Carlos ES Cesnik, and Hao Liu. "Recent progress in flapping wing aerodynamics and aeroelasticity." Progress in Aerospace Sciences 46, no. 7 (2010): 284327. https://doi.org/10.1016/i.paerosci.2010.01.001

[11] Dash, S. M., Kim-Boon Lua, T. T. Lim, and K. S. Yeo. "Enhanced thrust performance of a two dimensional elliptic airfoil at high flapping frequency in a forward flight." Journal of Fluids and Structures 76 (2018): 37-59. https://doi.org/10.1016/i.jfluidstructs.2017.08.008

[12] Yusoff, H., N. Iswadi, A. H. Zulkifly, Sh Mohd Firdaus, M. Z. Abdullah, M. K. Abdullah, and S. Suhaimi. "Lift performance of a cambered wing for aerodynamic performance enhancement of the flapping wing." Jurnal Teknologi 75, no. 8 (2015). https://doi.org/10.11113/it.v75.5208

[13] Song, Arnold, Xiaodong Tian, Emily Israeli, Ricardo Galvao, Kristin Bishop, Sharon Swartz, and Kenneth Breuer. "Aeromechanics of membrane wings with implications for animal flight." AIAA journal 46, no. 8 (2008): $2096-2106$. https://doi.org/10.2514/1.36694

[14] Kim, Dae-Kwan, and Jae-Hung Han. "Smart flapping wing using macrofiber composite actuators." In Smart structures and materials 2006: smart structures and integrated systems, vol. 6173, p. 61730F. International Society for Optics and Photonics, 2006. https://doi.org/10.1117/12.658117

[15] Shkarayev, Sergey, Dmitro Silin, Gregg Abate, and Roberto Albertani. "Aerodynamics of cambered membrane flapping wings." In 48th AIAA aerospace sciences meeting including the new horizons forum and aerospace exposition, p. 58. 2010. https://doi.org/10.2514/6.2010-58

[16] Ghommem, Mehdi, Muhammad Hajj, Layne Watson, Dean Mook, Richard Snyder, and Philip Beran. "Deterministic global optimization of flapping wing motion for micro air vehicles." In 13th AIAA/ISSMO Multidisciplinary Analysis Optimization Conference, p. 9355. 2010. https://doi.org/10.2514/6.2010-9355

[17] Rahman, Musfika, and Iskandar Dzulkarnain. "Response Surface Method for Modelling the Effect of CO2 in Brine/Waxy Oil Interfacial Tension during LSW-WAG Enhanced Oil Recovery." Journal of Advanced Research in Applied Sciences and Engineering Technology 22, no. 1 (2021): 54-68.

[18] M. R.H. and D. Montgomery (1995), Response surface methodology, U.S.A: John Wiley \& Sons, U.S.A. 